\title{
Contents Vol. 40, 1916
}

\author{
Monatsschrift
}

i'iir

\section{Psychiafrie und Neurologic}

Herausgegeben von

\section{K. Bonhoeffer,}

Bd. XL.

Mit zahlroielion AbbjMtuagen im Text und 6 Tafelo.

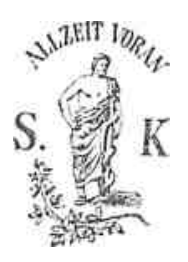

B E R L I N 1916

VERLAG VON S. KARGER.

KARLSTRASSE 15 
Alle Rechte vorbehalten.

Gcdruckt bei Imberg \& Lefson G. m. b. H. in Berlin SW 48 



\section{Inhalts - Verzeichnis.}

Original-Arbeiten.

Seite

JBonhoeffer, K., Zur psyohogenen Entwicklung und Hemmung kriegsneurotiscker Storungen. 199 Bumke, $H$., Die Beschleunigung der Blutgerinnungszeit bei Dementia piaecox................................................................ 344 Donath, Julius, Kriegsbeobachtungen liber hysterische Stimm-

Sprach- und Horstorungen 100

Forster, E., Isolierte Musculocutaneus-Lakmung bei Malaria . 262 Ganter, R., Uber die Behandlung der Epilepsie mit salzarmer

Kost und Sedobrol, und Sedobrol und Luminal. 378

Gerstmann, J., Zur Kenntnis der Storungen des Korpergleickgewichtes nack Scbufiverletzungen des Stirnkirns . . . 354 Kleist, K., Uber Leitungsapkasie und grammatiscke Storungen 118 Kutzinski, A., Apkasiscke Storungen nack gekauften epilepti-

seken Anfallen

—, - Einige Bemerkungen zur Psyckopatkologie der sogenannten Intestinal-Neurosen im AnsokluB an Erfahrungen bei Soldaten.

Pfeifer, A., Uber den feineren Bau des Zentralnervensystems eines Anencepkalus. (Hierzu Tafel I-VI) 1

Pick, A., Bewegung und Aufmerksamkeit. Ein Kapitel der allgemeinen Patkologie.

Schmidt, W., Sckizopkrenie und Dienstbesckadigung . . . 239 Schroder, P., Katatone Zustande bei progressiver Paralyse . . 30 Schwarz, G., Zur Kenntnis der Gedacktnisstorung nack Gra-

natenkontusion

Sauer, H., Uber gebaufte kleine Anfalle bei Eandern . . . 276 Severin, T., tlbei Adrenaknwirkung bei Sckizopkrenen und Gesunden 
Siebert, H., Uber die Tuberkub'nbehandlung der Paralyse . . 230

Weichbrodt, R., Eine einfache Liquorreaktion

Zangger, H., Erfahrungen bei einer Zelluloidkatastrophe. Mit besonderer Beriicksicbtigung der neurologiscben und psy-

chologiscben Gesicbtspunkte 88

Zimmermann, R., tJber den Alkaligehalt des Blutes bei Geistesgesunden und Geisteskranken

Ludwig Bruns f. 